\title{
Shape differences among symmetrically shaped skeletal growth patterns in a panoramic view: a Fourier analysis
}

\author{
Tania Camila \\ NIÑO-SANDOVAL ${ }^{(a)}$ iD \\ Marco FRAZÃO(b) iD \\ Belmiro Cavalcanti do Egito \\ VASCONCELOS(a) iD \\ (a) Universidade de Pernambuco - UPE, School \\ of Dentistry, Department of Oral and \\ Maxillofacial Surgery and Traumatology, \\ Recife, PE, Brazil. \\ (b)Faculdade de Odontologia do Recife - \\ FOR, Recife Dentistry College, Department \\ of Oral and Maxillofacial Radiology, Recife, \\ PE, Brazil.
}

Declaration of Interests: The authors certify that they have no commercial or associative interest that represents a conflict of interest in connection with the manuscript.

Corresponding Author:

Belmiro Cavalcanti do Egito Vasconcelos

E-mail: belmiro.vasconcelos@upe.br

https://doi.org/10.1590/1807-3107bor-2021.vol35.0034

Submitted: May 24, 2020

Accepted for publication: October 22, 2020

Last revision: November 27, 2020

\begin{abstract}
The objective of this study was to apply elliptic Fourier analysis (EFA) to find shape differences among skeletal growth patterns in both radiographic and tomographic panoramic views, controlling for asymmetry. Lateral and panoramic images were obtained from 350 patients. After screening patients with asymmetric linear and angular values and natural asymmetric hemimandibular shape, 240 patients were included in the study: 48 with tomographic information and 192 with radiographic information. The images were classified according to the mandibular plane angle and the ANB angle. Mandibular contours were digitized on the panoramic images and EFA was performed with 20 harmonics, filtering rotation, translation and size properties. As there were no differences between radiographic and tomographic panoramic mandibular contours and normal distribution was found in all groups, MANOVA was conducted to determine differences using a Hotelling's p-values with Bonferroni correction and an XY graph tool was applied to visualize these differences graphically. A 95\% confidence level was used. Significative differences were found among hypodivergent, normodivergent, and hyperdivergent patterns in Class I, II, and III ( $\mathrm{p}<0.05)$, located mainly in the symphyseal region. The results of this study suggest that EFA is a useful tool to mathematically analyze mandibular contours and their morphological differences given by facial biotypes. This method could improve the precision of the mandibular prediction models.
\end{abstract}

Keywords: Fourier Analysis; Mandible; Radiography, Panoramic; Tomography.

\section{Introduction}

Mandibular reconstruction systems offer few alternatives for resembling shape for the purposes of assisting in rehabilitation following mandibulectomy or forensic identification purposes. This is mainly related to the limitation of some studies to achieve an adequate analysis of the mandibular contour. One solution for obtaining more natural morphology resides in customized mathematical models. ${ }^{1,2,3}$ Although progress is evident compared to a few years ago, limitations are found in the scope of these systems for the general population.

Factors such as the cost and availability of resources in many places of difficult access exert an influence on this process. To establish an 
economical proposal for a mandibular prediction that is accessible to the entire Latin American population, bidimensional analyses are being extrapolated to a three-dimensional view. ${ }^{4,5,6}$ However, morphological components must be studied in depth to integrate the pertinent variables, enabling the determination of a mathematical model that can be generalized for the mass creation of models that resemble each property of mandibular bone, especially shape. For such purpose, elliptic Fourier analysis (EFA) could be employed. $7,8,9,10$

In facial reconstruction, it is relevant to highlight that the individuality of each person is directly related to its craniofacial biotype. ${ }^{11,12,13}$ For both maxillofacial surgery with rehabilitation purposes as for forensic identification, it is important to consider the best way to group the sample based on simple characteristics with morphological relevance. As craniofacial anatomy is highly complex, it is necessary to choose structural measures used in anthropology, orthodontics, and orthognathic treatment. Under these conditions, the combination of the ANB angle used to establish skeletal classes ${ }^{14}$ and the mandibular plane angle (S-N/ Go-Gn) used to establish skeletal growth patterns seems to be adequate to perform shape explorations. ${ }^{15}$

However, the study of skeletal growth patterns is mainly related to rotation and size factors of facial components. ${ }^{16}$ Besides, growth is generally measured in a lateral view by lines and angles and not by the shape given by the mandibular contour., ${ }^{41,13}$ As seen in previous studies, ${ }^{8,17}$ the evaluation of panoramic images allow an overview of the morphologic aspects of the mandibular contour. The use of EFA could give an idea of the specific regions that are characteristic of each biotype, allowing in the future the location of relevant landmarks that could be related to craniomaxillary angles for predictive purposes. ${ }^{4}$ As the explorations are performed in a panoramic view, linear, angular, and shape asymmetry should be controlled, as suggested previously by Niño-Sandoval. ${ }^{8}$

Thus, the aim of the present study was to apply EFA to find quantitative and qualitative shape differences among skeletal growth patterns in both radiographic and tomographic panoramic images, controlling for asymmetry.

\section{Methodology}

\section{Acquisition of images}

An observational exploratory research was carried out. Information from 350 patients was collected retrospectively from July 2017 to December 2018 at the Boris Berenstein Clinic in the city of Recife, Brazil. Fifty-four patients had tomographic information and 296 had information from lateral and panoramic radiographs. All patients were adults with full permanent dentition with no posterior crossbite, tumors, cysts, severe bone defects, congenital or acquired malformations, and no history of orthognathic or aesthetic surgery.

This study received approval from the Human Research Ethics Committee of the Dean's Office of Graduate Research and Innovation Programs of the University of Pernambuco (certificate number: 83767517.7.0000.5207) in the city of Recife and was conducted in accordance with the precepts stipulated in the Declaration of Helsinki.

Lateral radiographs were collected using a single X-ray device(ORTHOCEPH ${ }^{\circledR}$ OC200 D, Instrumentarium Dental; 60-85 kV; 3.2-16 mA; 8-20 s. Panoramic radiographs were taken using a single panoramic digital image system (ORTHOPANTOMOGRAPH ${ }^{\circledR}$ OP200 D, Instrumentarium Dental; $57-85 \mathrm{kV}$; $2-16 \mathrm{~mA}$; 14.1-17.6 s, Milwaukee, USA). The CLINIVIEW ${ }^{\mathrm{TM}}$ software (Instrumentarium Dental, Milwaukee, USA) was used for acquisition of radiographic data.

The I-CAT cone beam 3D dental imaging system was used for the tomograms (Version 3.1.62. Xoran Technologies, Ann Arbor, USA; and Imaging Sciences International, Hatfield, $\mathrm{PA} ; 360^{\circ}$ rotation; $120 \mathrm{kVp} ; 6 \mathrm{~mA}$; $0.30 \mathrm{~mm}$ pixel size; CT images: $768 \times 768 \times 576$, Ann Arbor, USA). The XoranCat ${ }^{\circledR}$ software (version 3.1.62. Xoran Technologies, Ann Arbor, USA; and Imaging Sciences International, Hatfield, USA) was used to visualize the images and to extract the lateral images.

To perform this task, the cutting plane lines were used to find the intermeatal plane in the axial view. Next, the slice thickness of the sagittal view was set to $75.3 \mathrm{~mm}$. The sagittal slice plane was first moved to the right and then to the left to confirm the correct alignment of the planes. The sagittal slice plane was then moved to the midline and the slice thickness was set to $150.3 \mathrm{~mm}$. For the panoramic view, the oblique 
tool was used to make a contour on the axial plane starting from the left meatus and ending with the right meatus, encompassing the condyles, sigmoid notch, coronoid process, teeth, mandibular body, and symphysis. Slice thickness was set to $20.1 \mathrm{~mm}$ to obtain a detailed image. Both images were adjusted to high resolution and exported to a.jpg format.

\section{Digitization of landmarks}

TPSUtil and TPSDig2 applications (Rohlf, F. J. 2005. Department of Ecology and Evolution, State University of New York at Stony Brook, New York, USA) were used to prepare the data and digitize the landmarks, hemimandibular curves, and total mandibular curves. Landmarks in lateral images were digitized as seen in Figure 1A and those in panoramic images were digitized as seen in Figure 1B. Three files were created for lateral images and three were created for panoramic images, each containing 350 images. The first and second files were used to perform the repeatability test with a three-week interval between the digitization of the two files.

The CLIC package was used to perform repeatability in panoramic and lateral images (Dujardi, Jean-Pierren, 2002, Institut de Recherches pour le Développement -
IRD, Montpellier, France). The MOG application was employed to perform a Procrustes analysis where the coordinate configurations of individuals are superimposed on a consensus, which corresponds to the average of all geometric configurations.

In this analysis, the properties of translation, scaling, and rotation were filtered. When translation was filtered, the geometric center of each individual shared the same centroid of the consensus. With those results, the size in each individual was scaled without losing its geometry. Then, those properties were submitted to the rotation filtering, where the homologous points of each configuration were adjusted to those points of the consensus configuration on the criterion of the least squares.

The results were submitted to partial warps and their principal components, the relative warps. Those outcomes were analyzed in the VAR function to compare the difference between both files in terms of the $X$ and $Y$ coordinates, using a Model II (or random effects) one-way ANOVA on repeated measures. ${ }^{18}$ The value of repeatability was provided by the ratio of the between-individual variance and the total variance. Considering these values, definitive landmarks were digitized in the remaining files.

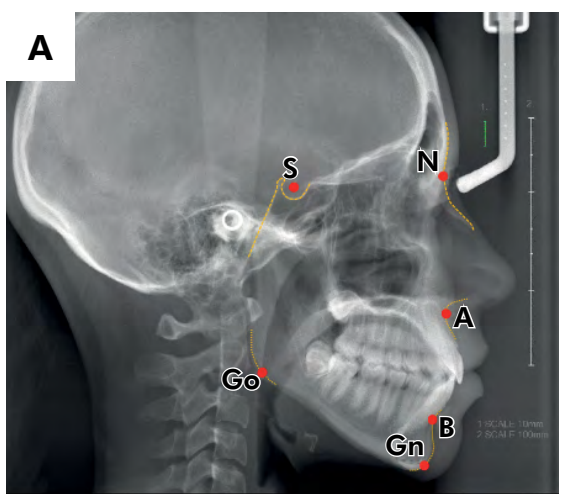

S: Midpoint of the sella turcica; N: Anterior point in the frontonasal suture; $\mathrm{A}$ : Deepest point of the anterior maxillaru contour; B: Deepest point of the anterior mandibular contour; Go: Intersection of the posterior border of the ramus to the inferior border of the mandibular body; Gn: Most anteroinferior point on the symphysis of the chin

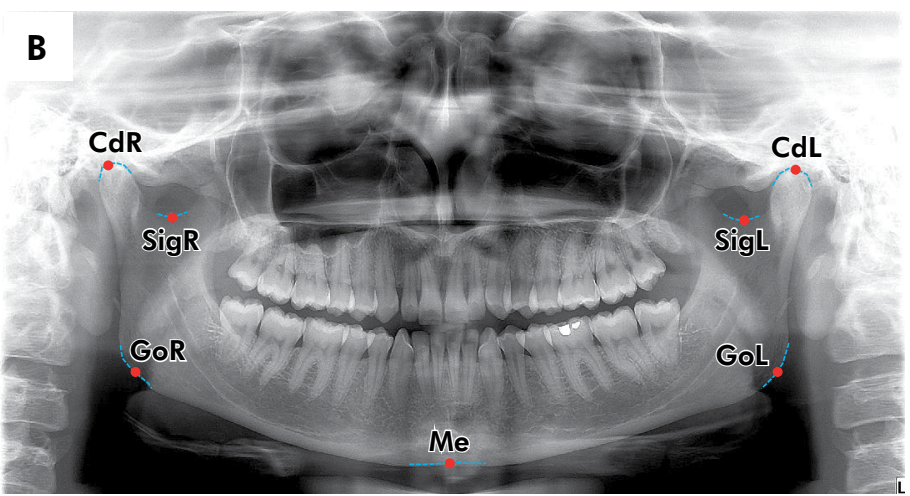

Cd: Most superior point on the condyle; Go: Intersection of the posterior border of the ramus to the inferior border of the mandibular body; Menton: Lowest point on mandibular symphysis; Sig: Deepest point of the sigmoid notch. R: Right; L: Left.

Figure 1. Landmarks location and definition. A. Landmarks in lateral cephalogram for determining ANB and S-N/MP angles. B. Landmarks in the panoramic view to evaluate asymmetry. 


\section{Digitization of curves}

For hemimandibular curves, a sagittal plane was traced to establish the facial midline, as described by Niño-Sandoval. ${ }^{8}$ The contours were then created using the "draw background curves" tool of the TPSDig2 software. In a closed contour, it is necessary to have the same starting point in all individuals in order to be comparable. Thus, the mandible needs to have an initial point that is easily identified and located. ${ }^{8,19,20}$ As the mandibular outline does not have any type 1 landmarks, such as sutures or edges, the landmark with the highest coefficient in the repeatability test was considered as the starting point. The right curve was drawn from the initial point. In another file, the left side was reflected to facilitate the asymmetry analysis and from the same initial point, the curve was drawn.

Likewise, the total mandibular contour was drawn from the same initial point for the entire sample. As the curves were closed contours, repeatability in this phase was not necessary. Each hemimandibular curve was re-sampled by length in 100 equidistant points and each total mandibular curve was re-sampled in 200 equidistant points, as made previously. ${ }^{7,8,21}$

\section{Control of asymmetry and final selection of patients}

MATLAB $^{\circledast}$ (R2017b - MathWorks, 2017, Natick, USA) was used to calculate linear and angular measures from $X$ and $Y$ coordinates. From the panoramic view, left and right mandibular ramus height (CdL-GoL and CdR-GoR), left and right mandibular body size (GoL-Me and GoR-Me), left and right gonial angle (CdL-GoL-Me and CoR-GoR-Me), and distance between left and right condyles (CdL and $\mathrm{CdR})$ to the menton $(\mathrm{Me})$ were calculated.

A comparison was made by using a t-test, taking into account the right side and the left side as two independent samples. To perform this comparison, as an initial exploration, individuals that had differences between left and right sides greater than $5 \mathrm{~mm}$ and $5^{\circ}$, were excluded. These measures were adjusted until significant differences were no longer obtained.

The Morpho J program was used to perform the shape asymmetry tests on the selected patients. ${ }^{22}$ The 'find outliers' function was applied. This tool allows to visualize and exclude those individuals that are outside the expected mean shape of the whole sample. From this average, the cumulative distribution of the distances of each individual included was considered.

A Procrustes fit analysis was used for the remaining sample. In this analysis, size, position, and rotation were filtered, leaving only the shape property. An ANOVA was applied to compare panoramic shapes digitized in tomographic and radiographic sources in order to unify the contours. Once there were no significant differences between the sources of data collection, another ANOVA was used to compare the right and left sides to confirm the general symmetry of the samples.

\section{Grouping of patients}

With Matlab, the SNA, SNB, ANB, and mandibular plane angles were calculated in the selected patients. An ANB angle between $1^{\circ}$ and $3^{\circ}$ was considered Class I; an ANB angle $\geq 4^{\circ}$ was considered Class II, and negative angles obtained by subtracting SNA from SNB were considered Class III. The mandibular plane angle was measured and the hypodivergent pattern was established if the S-N/MP angle was $<28^{\circ}$; a normodivergent pattern was established when the angle was between $28^{\circ}$ and $36^{\circ}$, and a hyperdivergent pattern was established when the S-N/MP angle was $>36^{\circ} .{ }^{15}$

\section{Panoramic shape differences}

The PAST software (Hammer, $\varnothing$, Harper, D.A.T, Ryan, P.D. 2013. PAST: Paleontological Statistics software package for education and data analysis, Version 2.17c, Oslo, Norway ) was employed to quantify differences between hyperdivergent, normodivergent, and hypodivergent skeletal growth patterns in patients categorized in Classes I, II, and III by using EFA.

EFA is a tool that decomposes each coordinate that conforms a contour into cosine and sine functions. ${ }^{79,10}$ It is applied when a figure placed on a Cartesian plane is in a relationship in which a coordinate on the $\mathrm{X}$-axis has two or more values on the Y-axis (Figure 2A). Each set of functions is seen as an ellipse called harmonic that encompasses the greatest number of points on the curve. ${ }^{10}$ A greater number of harmonics leads to a better definition of the shape. However, 
when there is no difference between harmonics, it is important to establish a limit to avoid redundant data. ${ }^{8}$ Considering this, twenty elliptic Fourier harmonics with the same starting point were chosen (Figure 2B).

Direct shape comparisons between different biotypes are possible by filtering scale, translation, and rotation properties. The scaling is defined as the size property of the contour. The greatest variation in shape comparisons is related to the differences in the magnitude of the contours, especially if they came from different sources of collection, such as tomograms and radiographs (Figure 2C). EFA removes the differences from the data by reciprocal scaling, that is, the contours are re-scaled to eliminate size differences among a set of outlines. ${ }^{8,10,23}$

The translation describes the displacement of each point of the figure in the same direction, it is a property that also influences the variation. This is because the contours could be located in different regions of the cartesian plane, hence the variation is greater if the distances between the points of the contours are greater. Rotation, on the other hand, is a movement from one coordinate system to another in a given space that retains at least one point in its original position. ${ }^{23}$

To control both location properties, EFA considers the starting point, helping in the alignment of the other harmonics set by the first harmonic. After that, the model coefficients obtained from EFA are adjusted into a standing rotational orientation, aligning all the specimens in the same position (Figure 2D). When that happens, the $\mathrm{X}$ cosine of the first harmonic corresponds to $1 .^{10,23}$ The data obtained from the harmonics by using EFA was processed by a principal component analysis (PCA). A Mardia multivariate normality test was employed to determine whether a parametric MANOVA or non-parametric MANOVA should be applied to evaluate shape variations and their distinctiveness. ${ }^{23} \mathrm{~A}$ confusion matrix tool of the MANOVA was employed to analyze the discriminatory capacity of the model. The main value is determined by the accuracy, defined as the proportion of correct classifications among all classifications. Values $\geq 80 \%$ were considered excellent accuracy, $<80 \%$ to $70 \%$ was considered good accuracy, $<70 \%$ to $60 \%$ was considered moderate accuracy, $<60 \%$ to $50 \%$ was considered mild accuracy, and $<50 \%$ was considered low accuracy.

For the qualitative analysis, Tpsrelw32 (Version 1.65. Rohlf, F. J. 2016. Department of Ecology and Evolution, State University of New York at Stony Brook, New York, USA) was used to determine the mean shape of each group. These data were extrapolated to PAST and the ' $X Y$ graph' tool was applied to visualize the differences among the skeletal growth patterns. This graphic was a visual aid to indicate the specific places where differences were found. A qualitative description of the differences in hypodivergent and hyperdivergent growth patterns compared to the normodivergent pattern was given.

\section{Results}

Table 1 shows the results for repeatability by coordinates of each landmark. Coefficients closer to 1 were observed, indicating a low variation in the location of points. Considering that the highest values for both coordinates in the panoramic view were obtained in the right sigmoid notch, this landmark was designated as the starting point of the mandibular contour.

In the linear and angular symmetry selection of the sample, p-values of $0.97,0.48,0.84$, and 0.34 were obtained for the mandibular height, mandibular body, the distance between $\mathrm{Cd}$ and $\mathrm{Me}$, and the gonial angle, respectively. The mean differences of those measures were of $\approx 1.58 \mathrm{~mm}$ for the mandibular height, $\approx 1.98 \mathrm{~mm}$ for the mandibular body, $\approx 1.72 \mathrm{~mm}$ for the distance between condylion to the menton, and $\approx 2.05^{\circ}$ for the gonial angle.

Considering this, panoramic radiographs of 229 patients and tomograms of 49 patients were selected for the next phase of exploration of the sample distribution. Accordingly, with the distribution parameters related to the expected mean shape, the 'find outliers' function indicated the additional exclusion of one tomographic sample and 37 panoramic radiographs.

Subsequently, shape explorations were carried out. Shape differences between tomographic and panoramic contours were not significant $(p=0.735)$, allowing the unification of the contours obtained by 


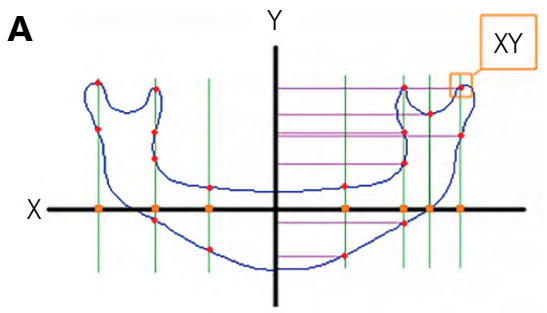

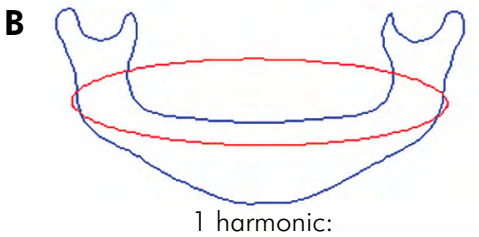

Cosx_1 Sinx_1 Cosy_1 Siny_1

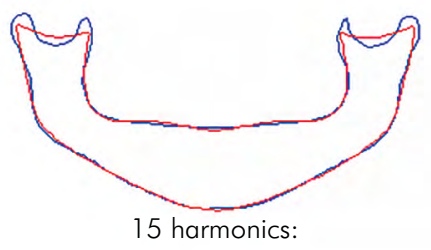

Cosx_15 Sinx_15 Cosy_15 Siny_15

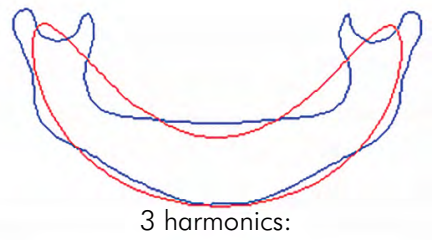

Cosx_3 Sinx_3 Cosy_3 Siny_3

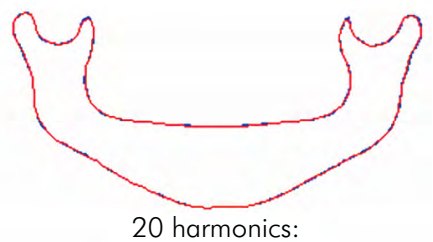

Cosx_20 Sinx_20 Cosy_20 Siny_20
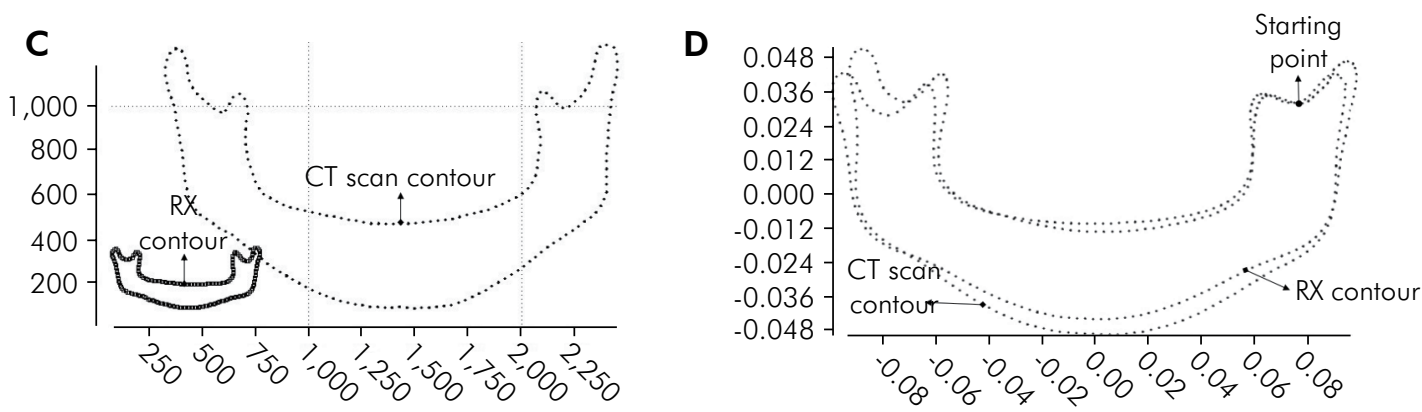

Figure 2. Example of the mandibular contour processing through an Elliptic Fourier Analysis (EFA). A. Mandibular contour placed in a Cartesian plane: when a value in X-axis has two or more values in Y-axis, a relationship is established. B. To avoid statistical problems, those relationships are transformed with EFA into sine and cosine functions to form 20 harmonics reconstructing the mandibular curvature. C. An example of raw data of two panoramic contours from two different collection sources: size and position determine the main differences. D. EFA controlling for size, translation, and rotation aligning the samples from the same starting point, so the shape can be directly compared.

Table 1. Landmarks repeatability evaluation using randomeffects one-way ANOVA.

\begin{tabular}{lccc}
\hline View & Landmark & X coordinate & Y coordinate \\
\hline \multirow{4}{*}{ Lateral } & $\mathrm{S}$ & 0.94 & 0.97 \\
$\mathrm{~N}$ & 0.90 & 0.96 \\
& $\mathrm{~A}$ & 0.95 & 0.96 \\
& $\mathrm{~B}$ & 0.92 & 0.93 \\
& $\mathrm{Go}$ & 0.95 & 0.95 \\
& $\mathrm{Gn}$ & 0.94 & 0.93 \\
\hline \multirow{4}{*}{ Panoramic } & $\mathrm{CdR}$ & 0.96 & 0.92 \\
& $\mathrm{CdL}$ & 0.93 & 0.92 \\
& $\mathrm{SigR}$ & 0.99 & 0.98 \\
& $\mathrm{SigL}$ & 0.98 & 0.97 \\
& $\mathrm{GoR}$ & 0.93 & 0.93 \\
& $\mathrm{GoL}$ & 0.95 & 0.93 \\
& $\mathrm{Me}$ & 0.92 & 0.93 \\
\hline
\end{tabular}

Closest values to 1.0 show better repeatability by coordinates. different collection sources. In addition, no significant difference was obtained when comparing the left and right shapes $(p=0.8302)$, confirming the control of natural asymmetry in the selected sample.

After those analyses, the definitive sample was composed of 240 patients: 48 with tomographic information and 192 with radiographic information. Table 2 shows the biotypes classification of those patients, defining facial biotypes as the union of skeletal classifications with the skeletal growth patterns. The mean and standard deviation of the ANB angles were determined, and it can be seen that the data extend in a wider range of values, especially in group 6 that corresponds to hyperdivergent class II and group 9 that corresponds to hyperdivergent Class III.

In groups 1, 2, and 3 that correspond to hypodivergent, normodivergent, and hyperdivergent 
Table 2. Classification by skeletal classes and skeletal growth patterns in selected patients after the asymmetry filtering process.

\begin{tabular}{|c|c|c|c|c|c|c|c|c|c|c|c|}
\hline \multirow{2}{*}{ Group } & \multicolumn{2}{|c|}{ ANB angle } & \multicolumn{7}{|c|}{ S-N/MP } & \multicolumn{2}{|c|}{ Sex } \\
\hline & Mean & SD & Skeletal class & Mean & $\mathrm{SD}$ & 25\% Quantile & Median & 75\% Quantile & Skeletal growth pattern & $\mathrm{F}$ & M \\
\hline 1 & 2.47 & 0.7 & \multirow{3}{*}{ । } & 23.83 & 3.6 & 22.51 & 24.73 & 26.34 & Hypodivergent & 5 & 12 \\
\hline 2 & 2.59 & 0.73 & & 31.73 & 2.38 & 29.63 & 31.5 & 34 & Normodivergent & 33 & 22 \\
\hline 3 & 2.37 & 0.74 & & 39.21 & 2.67 & 37.23 & 38.15 & 39.83 & Hyperdivergent & 10 & 9 \\
\hline 4 & 5.03 & 0.89 & & 23.79 & 3.63 & 23.14 & 24.89 & 26.39 & Hypodivergent & 7 & 10 \\
\hline 5 & 5.79 & 1.55 & & 32.81 & 2.47 & 30.5 & 32.59 & 34 & Normodivergent & 22 & 15 \\
\hline 6 & 6.94 & 2.11 & & 41.81 & 4.99 & 38.22 & 39.7 & 43.82 & Hyperdivergent & 32 & 7 \\
\hline 7 & -2.03 & 0.73 & \multirow{3}{*}{ III } & 22.58 & 3.54 & 18.84 & 23.55 & 25.26 & Hypodivergent & 5 & 10 \\
\hline 8 & -1.9 & 0.9 & & 31.59 & 2.26 & 29.51 & 30.87 & 32.79 & Normodivergent & 14 & 17 \\
\hline 9 & -1.41 & 1.4 & & 40.23 & 2.97 & 38.09 & 39.39 & 41.83 & Hyperdivergent & 4 & 6 \\
\hline
\end{tabular}

patterns of the skeletal Class I, and groups 4 and 7 that correspond to the hypodivergent pattern of class II and III respectively, a more centralized dispersion in ANB angles were obtained. On the other hand, groups 5 and 8 that correspond to normodivergent patterns of class II and class III presented values with broader ranges.

In reference to the classification by skeletal patterns, due to the ordinal nature of the data and greater standard deviations, a quantile analysis was performed. In general, the median values were close to those of the mean, especially in groups 2 and 5 . The furthest median value from the mean occurred in group 6. The widest interquartile ranges were found in groups 6 and 7, and the lowest was found in group 3 . The distribution by sex was generally unbalanced, especially in group 6. Due to this imbalance, no tests for sexual dimorphism were carried out.

Quantitative comparisons between all skeletal growth patterns in Classes I, II, and III were made with the first 10 axes of the PCA applied to the 20 harmonics that explained $94.58 \%$ of the variance. Figure 3 shows the principal component analysis scatter diagram. Convex hulls were used to facilitate the visualization of the distributions of the sample. A centralized distribution of the normodivergent pattern that overlaps the other skeletal patterns was observed in the skeletal Class I group (Figure 3A) and the skeletal Class III group (Figure 3C). Also, in reference to the main axis in both class I and III groups, hypodivergent patterns tended to the positive side and the hyperdivergent group tended to the negative side. Despite being on opposite sides, the hypodivergent and hyperdivergent patterns partially overlapped in the center of the two axes. Referring to Class II, the distribution of the groups remained similar, overlapping in the central part of the first two axes.

The Mardia multivariate normality test gave a value of 0.1569 , following a normal distribution, hence a parametric MANOVA was applied and its results are shown in Table 3. In this analysis, two statistical tests were performed to assess whether the means of the variables differed between the groups. The first test, which is the Wilks' Lambda, indicated significant differences in the three skeletal classes. The Pillai's trace test was also performed to find significant differences. Both tests rejected the null hypothesis of no differences between groups. To find out specifically which groups determined those significant differences, pairwise comparisons by skeletal patterns using Hotelling's $\mathrm{p}$ values, corrected by Bonferroni test, were done. The results showed a statistically significant difference between all the skeletal patterns in Class I, II, and III.

Figure 4 shows the mandibular regions where the main differences between skeletal patterns by class were concentrated. The zone with the greatest variation was located mainly in the mandibular inferior border in Class I, II, and III (Figures 4A, 4B, and $4 \mathrm{C}$, respectively).

Considering the normodivergent pattern (blue) as a reference, in all three skeletal classes, the hypodivergent pattern (beige) was directed upward and the hyperdivergent pattern (red) was directed downward, especially in the symphyseal 

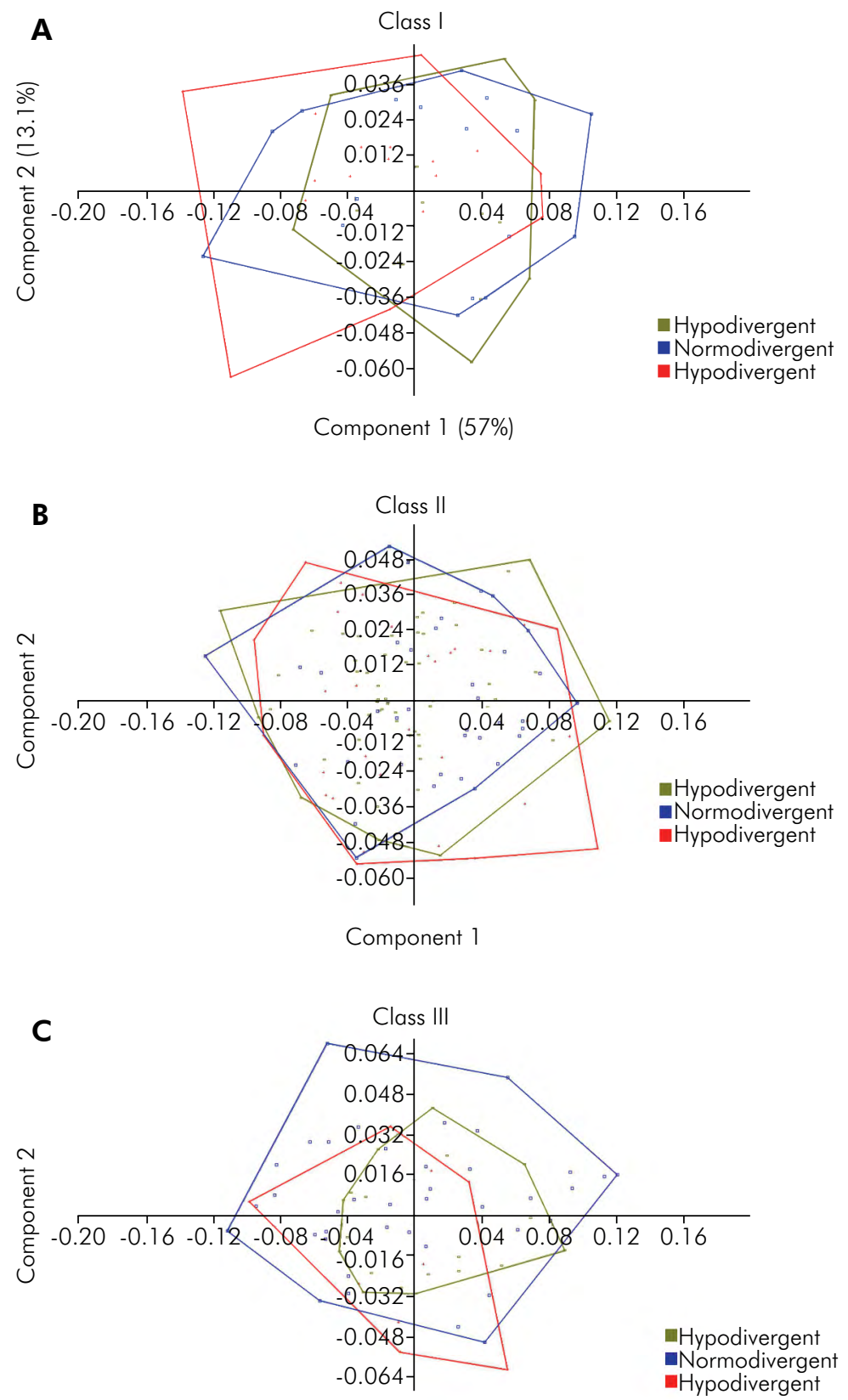

Component 1

Figure 3. Scatter diagram with convex hulls of the principal component analysis (PCA) for the skeletal growth patterns in each ANB class, defined by the first two principal components. A. Scatter diagram in Class I. B. Scatter diagram in Class II. C. Scatter diagram in Class III.

contour. A similar distribution was observed in the alveolar zone, primarily in Class I and Class II (Figures 4A and 4B); in Class III, the normodivergent and hyperdivergent patterns were very similar (Figure 4C). In the gonial angles, it was noteworthy that the hyperdivergent pattern presented angles that were more open, being consistent with a more elongated morphology, specifically in Class I (Figure 4A) and Class III (Figure 4C). Table 4 shows a confusion matrix of the MANOVA results. Accuracy in Class I was of $73.6 \%$, in Class II, $70.9 \%$, and in Class III, $76.7 \%$ 
Table 3. Comparison of mandible mean shapes between skeletal growth patterns in Class I, II, and III, by MANOVA. Significant differences are considered when $p<0.05$.

\begin{tabular}{|c|c|c|c|c|c|}
\hline \multirow{2}{*}{ Variable } & \multirow{2}{*}{ Value } & \multirow{2}{*}{ Variable } & \multirow{2}{*}{ Value } & \multicolumn{2}{|l|}{ Pairwise comparisons* } \\
\hline & & & & Skeletal growth patterns & $p$-values \\
\hline \multicolumn{6}{|l|}{ Class I } \\
\hline Wilks' Lambda & 0.64 & Pillai trace & 0.37 & & \\
\hline $\mathrm{dfl}$ & 2 & df 1 & 2 & Hypodivergent vs Normodivergent & $4.61 \mathrm{E}-06$ \\
\hline$d f 2$ & 88 & $d f 2$ & 88 & \multirow{2}{*}{ Hypodivergent vs Hyperdivergent } & \multirow{2}{*}{ 5.05E-06 } \\
\hline $\mathrm{F}$ & 24.05 & F & 26.93 & & \\
\hline $\mathrm{p}($ same $)$ & $4.65 \mathrm{E}-09$ & $\mathrm{p}($ same $)$ & $7.51 \mathrm{E}-10$ & Normodivergent vs Hyperdivergent & 0.003 \\
\hline \multicolumn{6}{|l|}{ Class II } \\
\hline Wilks' Lambda & 0.69 & Pillai trace & 0.31 & & \\
\hline$d f 1$ & 2 & $d f 1$ & 2 & Hypodivergent vs Normodivergent & 0.02 \\
\hline$d f 2$ & 90 & $d f 2$ & 90 & \multirow{2}{*}{ Hypodivergent vs Hyperdivergent } & \multirow{2}{*}{$8.86 \mathrm{E}-06$} \\
\hline $\mathrm{F}$ & 19.65 & F & 21.05 & & \\
\hline $\mathrm{p}($ same $)$ & $8.29 E-08$ & $\mathrm{p}($ same) & $3.1 \mathrm{E}-08$ & Normodivergent vs Hyperdivergent & $8.35 \mathrm{E}-05$ \\
\hline \multicolumn{6}{|l|}{ Class III } \\
\hline Wilks' Lambda & 0.53 & Pillai trace & 0.53 & & \\
\hline $\mathrm{df1}$ & 2 & df 1 & 2 & Hypodivergent vs Normodivergent & $6.18 \mathrm{E}-05$ \\
\hline$d f 2$ & 53 & $d f 2$ & 53 & \multirow{2}{*}{ Hypodivergent vs Hyperdivergent } & \multirow{2}{*}{0.0002} \\
\hline $\mathrm{F}$ & 23.08 & F & 30.02 & & \\
\hline $\mathrm{p}($ same $)$ & $6.1 \mathrm{E}-08$ & $\mathrm{p}($ same $)$ & $1.91 \mathrm{E}-09$ & Normodivergent vs Hyperdivergent & 0.002 \\
\hline
\end{tabular}

*Hotelling's $p$ values, corrected by Bonferroni post-hoc test.

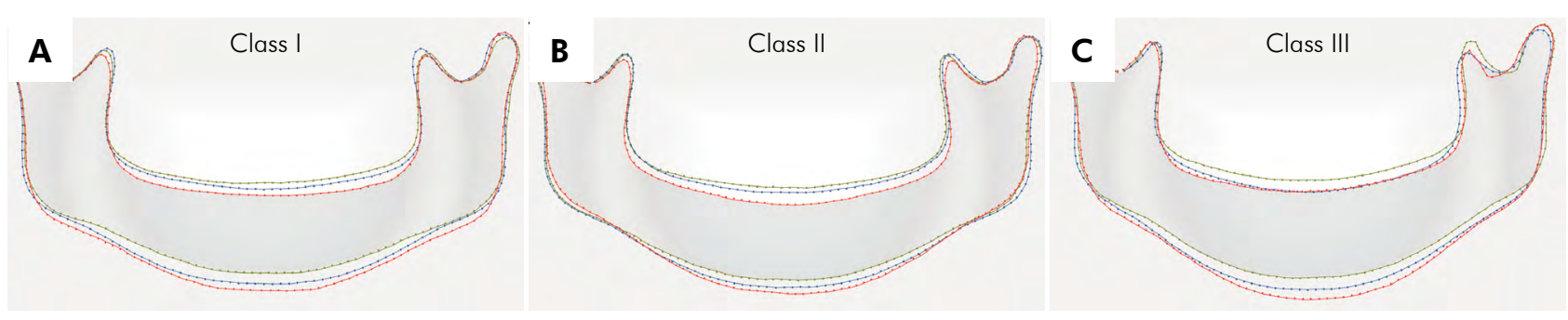

Figure 4. Differences in mean shape between hypodivergent (beige), normodivergent (blue), and hyperdivergent (red) skeletal growth patterns. A. Differences in Class I. B. Differences in Class II. C. Differences in Class III.

\section{Discussion}

The present study offers the quantification of a complex geometric figure. The mandible is a composition of several curves that differ significantly among growth patterns in each skeletal class. The geometric contour is essential to establish these differences graphically, and the results of the EFA enabled obtaining mathematical values that can be compared.

The mandibular measures have been previously highlighted as output variables in different classification model. ${ }^{4,5,6}$ The relationships between craniofacial variables have obtained satisfactory results, however, the mandibular curvature and its characteristic shape for each biotype have not been explored as output variables, especially in panoramic shape. In this exploration, it was possible to understand that the mandibular shape can be related to each biotype determined with angles in lateral images, giving an important link between sagittal and panoramic views. This association highlights the importance of geometric morphometric analysis like EFA, and that shape is a property that must be considered a variable as important as size and position. 
Table 4. Confusion matrix for mandibular shape in Class I, II, and III.

\begin{tabular}{|c|c|c|c|c|}
\hline Variable & True hypodivergent & True normodivergent & True hyperdivergent & Class precision \\
\hline \multicolumn{5}{|c|}{ Accuracy Class I: $73.6 \%$} \\
\hline Hypodivergent & 11 & 4 & 2 & $64.7 \%$ \\
\hline Normodivergent & 7 & 42 & 6 & $76.3 \%$ \\
\hline Hyperdivergent & 2 & 3 & 14 & $73.6 \%$ \\
\hline Class Recall & $55 \%$ & $85.7 \%$ & $63.6 \%$ & \\
\hline \multicolumn{5}{|c|}{ Accuracy Class II: 70.9\% } \\
\hline Hypodivergent & 13 & 3 & 1 & $76.4 \%$ \\
\hline Normodivergent & 4 & 28 & 5 & $75.6 \%$ \\
\hline Hyperdivergent & 8 & 6 & 25 & $64.1 \%$ \\
\hline Class Recall & $52 \%$ & $75.6 \%$ & $80.6 \%$ & \\
\hline \multicolumn{5}{|c|}{ Accuracy Class III: 76.7\% } \\
\hline Hypodivergent & 12 & 2 & 1 & $80 \%$ \\
\hline Normodivergent & 4 & 23 & 4 & $74.1 \%$ \\
\hline Hyperdivergent & 0 & 2 & 8 & $80 \%$ \\
\hline Class Recall & $75 \%$ & $85.1 \%$ & $61 \%$ & \\
\hline
\end{tabular}

The common finding in the shape analysis of the three skeletal classes was the fact that the greatest variation was located in the symphyseal zone. This is in agreement with data described by Ajmera et al., ${ }^{13}$ who found that symphyseal morphology, especially the symphyseal angle, had discriminatory power in determining skeletal patterns in panoramic radiographs. In the present study, an upward and more horizontal shape of the mandibular body was found in the hypodivergent pattern. Conversely, a V-shaped symphyseal zone was found in the hyperdivergent pattern.

The normodivergent pattern was located between the hyperdivergent and hypodivergent patterns in the middle zone of the mandible. The importance of this result is the discriminatory capacity found in this specific area, which is linked to the lateral view. ${ }^{17}$ This enables connecting certain anatomical points, thereby facilitating the three-dimensional characterization. Thus, special attention should be given to this anatomical zone in future investigations.

Despite similarities in the condylar anatomy between skeletal patterns, it is important to mention that shape analyses are difficult to perform in this zone due to its small size and numerous details. To adapt this structure equally to the general contour, it is necessary to soften many of its characteristics. Anticipating this situation, 200 points were used for each mandibular contour, thereby minimizing possible losses in its anatomy. However, it is highly recommended to perform an additional specific condylar analysis to confirm these shape differences.

Regarding shape in the gonial region, the differences occurred mainly in the hyperdivergent pattern. According to some researchers, the gonial zone in the posteroanterior and lateral views is very important and offers high accuracy in prediction tasks. ${ }^{4,6,24}$ Although the differences were not as great as those in the symphyseal zone, the differences found in the present study could have higher discriminatory power in lateral cephalograms and tomograms, which would facilitate prediction tasks.

One of the most variable areas was the coronoid process. This zone presented a high degree of differences between the individuals of the same group, observable in the distribution with the Morpho J program. This situation was reflected in the mean shapes (Figure 4), mainly in the hypodivergent pattern in Class I and Class II, and in all three patterns in Class III. Despite this situation, this structure was not relevant in the general analysis, since it did not affect the other mandibular regions. In mandibular morphology studies, this area is usually not contemplated for reconstruction purposes, and it is sometimes considered only for graphic purposes but after that, it is modified or removed. ${ }^{25,26,27}$ 
Regarding the confusion matrix tool results, in the three skeletal classes, the class precision in the hyperdivergent and hypodivergent patterns are mainly influenced by those normodivergent values considered as positive. Likewise, in the normodivergent pattern, the distribution of misclassified values between hypodivergent and hyperdivergent are similar. In the class recall, the proportion of correctly classified values also decreases due to the influence of false negatives given mainly by the misclassification of the normodivergent pattern.

This is explained by the fact that the normodivergent pattern is located among a spectrum of morphologies where one end is identified with hyperdivergent morphology and the other one is identified with hypodivergent morphology. This would also explain the trends of the PCA scatter plot, especially in Class I and III. This could be reflected also in the intermediate morphology of the normodivergent pattern between the other two patterns, especially in the symphysis (Figures 4A and C).

The only exception was found in Class II for which the main influence on the lower classification values of the hypodivergent pattern was given by the hyperdivergent pattern. In this class, there was also an important overlap between these opposite classes with the normodivergent pattern in the PCA scatter plot. This could be explained by the influence of the data of the group 6, (class II normodivergent pattern) that had the widest interquartile ranges and the greatest standard deviations in both ANB and S-N/ MP angles. These ranges possibly prevented greater discrimination of the model, even if the differences were significant. Figure $4 \mathrm{~B}$ shows that although differences among patterns are observable they are not as remarkable as in the other classes.

One of the essential aspects of this study was the control for asymmetry and all preprocessing steps. ${ }^{28}$ With the initial check of the linear and angular measurements and the control of the natural asymmetry of the mandibular shape, it was possible to exclude samples that would skew the results of the general shape outcomes. The panoramic view favored this process, with advantages over other mandibular views by assisting in the study of the mandibular asymmetries. The panoramic view also encompasses the entire mandibular morphology, enabling a description of the natural contour. ${ }^{8,28}$

Moreover, EFA enabled controlling for the unequal distribution of the sample. As the 'find outliers' tool of MorphoJ software was applied, the distances between the individuals and the mean shape were lower, hence, the sample was more uniform. When the sample was submitted to this tool by group distribution, a general low variation was also observed. In this case, when future patients fall within the average of each group, they are expected to not alter this consensus significantly and the results will be similar regardless of the number of the patients in each group. On the other hand, with EFA, after determining the mean shape, the number of patients included in a group is not a variable that modifies the results, therefore the imbalance between the groups was not relevant in the statistical analyses.

Despite this, the distribution of patients in the present study limited other possible explorations and the creation of more specific groups that integrate other variables. An example of this was the inability to perform a sexual dimorphism test, which was significantly relevant to the panoramic shape in a Colombian sample in a previous study. ${ }^{8}$ Other variables of considerable interest to the diagnosis, such as jaw size, molar and canine relation, and/or sub-classifications given by the SNA and SNB angles, also had to be excluded due to the lack of a sufficient sample size in each group.

However, it is important to point out that controlling natural asymmetry led to the exclusion of $31.4 \%$ of the initial sample in the present study. Without this control, the data would not have been as precise and the differences in both numerical values and graphs would not have been accurate. Therefore, it is necessary to have a larger universe without apparent asymmetries to increase the probability of choosing a more symmetric sample that does not affect morphometric outcomes.

Moreover, the processing of contours obtained from radiographs and tomograms indicated that the dissimilarities between different types of images were controlled by filtering size and rotation characteristics, leaving only the most essential feature of mandibular configuration (shape). With this data, 
the extrapolation of this two-dimensional information to three-dimensional models could be facilitated.

Previous studies of the same line of research have observed how lines and angles represented by dimensional values and radians values respectively can be related to craniomaxillary angles in predictive models. ${ }^{4,6}$ Taking this idea into account, it is possible in future approaches to relate craniomaxillary angles with the mandibular values corresponding to the sines and cosines obtained from EFA, or other methods provided by Geometric Morphometrics, for the creation of predictive models.

Although in this exploration the size, translation, and rotation factors were filtered to only compare shape, the latter proved to be a descriptive and distinctive property of mandibular morphology and should be considered as important as the other traditional variables. Another advantage of EFA is related to the generation of a consensus, which is the mean shape of a set of figures. Table 2 shows nine biotypes, each one constituted by an unbalanced sample; however, by averaging the mandibular contours of a group, a consensus per biotype is created, and then it can be compared with another consensus quantitatively and qualitatively. This is very useful, especially when the prevalence of the skeletal patterns varies in different populations.

In this article, some aspects should be considered to continue the explorations for the creation of three-dimensional models. The first is that it is possible to reduce the differences between the contours obtained between various sources of data (tomograms and radiographs) which can facilitate the extrapolation of two-dimensional data to three-dimensional approaches.

The second aspect is related to the achievement of results that can contemplate various facial biotypes in populations. We showed that the combination of Angle's skeletal classes and skeletal growth patterns must be considered. These have significant discriminatory power and little intergroup variability (with the aforementioned exception of the coronoid process).

The third aspect to consider is that the normalization of the sample is accompanied by the control of the mandibular asymmetry. This control should not only be done in the linear and angular measures, but also in the general shape contour of the mandible. This is important to be applied in the definitive sample of the three-dimensional prediction model to avoid an undesirable effect in the graphic results.

Future studies should perform this analysis in the lateral view for a comparison to the data obtained in the present investigation and find the best discriminant variables to facilitate the extrapolation of the information to a three-dimensional view. Moreover, the control of the natural asymmetry in the mandibular curvature should always be taken into consideration when including new patients in this model

\section{Conclusion}

The present study demonstrated that EFA is an effective tool that can find quantitative and significant shape differences among skeletal growth patterns in panoramic views, controlling for asymmetry. These differences are located mainly in the mandibular symphysis.

\section{Acknowledgments}

The authors are grateful to the Brazilian funding agencies Coordenação de Aperfeiçoamento de Pessoal de Nivel Superior (CAPES [Coordination for the Advancement of Higher Education Personnel]) and Conselho Nacional de Desenvolvimento Científico e Tecnológico (CNPq [National Council of Scientific and Technological Development]).

\section{References}

1. Wang Y, Zhang Y, Zhang Z, Li X, Pan J, Li J. Reconstruction of Mandibular Contour Using Individualized High-Density Porous Polyethylene (Medpor ${ }^{\circledR}$ ) implants under the guidance of virtual surgical planning and 3D-Printed surgical templates. Aesthetic Plast Surg. 2018 Feb;42(1):118-25. https://doi.org/10.1007/s00266-017-1029-2 
2. Kraeima J, Glas HH, Witjes MJ, Schepman KP. Patient-specific pre-contouring of osteosynthesis plates for mandibular reconstruction: using a three-dimensional key printed solution. J Craniomaxillofac Surg. 2018 Jun;46(6):1037-40. https://doi.org/10.1016/j.jcms.2018.03.022

3. Benazzi S, Fantini M, De Crescenzio F, Mallegni G, Mallegni F, Persiani F et al. The face of the poet Dante Alighieri reconstructed by virtual modelling and forensic anthropology techniques. J Archaeol Sci. 2009;36(2):278-83. https://doi.org/10.1016/i.jas.2008.09.006

4. Niño-Sandoval TC, Guevara Pérez SV, González FA, Jaque RA, Infante-Contreras C. Use of automated learning techniques for predicting mandibular morphology in skeletal class I, II and III. Forensic Sci Int. 2017 Dec;281:187.e1-7. https://doi.org/10.1016/i.forsciint.2017.10.004

5. Niño-Sandoval TC, Guevara Perez SV, González FA, Jaque RA, Infante-Contreras C. An automatic method for skeletal patterns classification using craniomaxillary variables on a Colombian population. Forensic Sci Int. 2016 Apr;261:159.el-6. https://doi.org/10.1016/j.forsciint.2015.12.025

6. Niño-Sandoval TC, Guevara Pérez SV, González FA, Jaque RA, Infante-Contreras C. [Use of artificial neural networks for mandibular morphology prediction through craniomaxillar variables in a póstero-anterior view]. Univ Odontol. 2016;35(74):21-8. Spanish. https://doi.org/10.11144/Javeriana.vo35-74.urna

7. Caple JM, Byrd JE, Stephan CN. The utility of elliptical Fourier analysis for estimating ancestry and sex from lateral skull photographs. Forensic Sci Int. 2018 Aug;289:352-62. https://doi.org/10.1016/j.forsciint.2018.06.009

8. Niño-Sandoval TC, Morantes Ariza CF, Infante-Contreras C, Vasconcelos BC. Evaluation of natural mandibular shape asymmetry: an approach by using elliptical Fourier analysis. Dentomaxillofac Radiol. 2018 Jul;47(6):20170345. https://doi.org/10.1259/dmfr.20170345

9. Lestrel PE, Wolfe CA, Bodt A. Mandibular shape analysis in fossil hominins: fourier descriptors in norma lateralis. Homo. 2013 Aug;64(4):247-72. https://doi.org/10.1016/i.jchb.2013.05.001

10. Chen SY, Lestrel PE, Kerr WJ, McColl JH. Describing shape changes in the human mandible using elliptical Fourier functions. Eur J Orthod. 2000 Jun;22(3):205-16. https://doi.org/10.1093/ejo/22.3.205

11. Khan MY, Kishore MS, Bukhari SA, Rachala MR, Sashidhar NR. Alveolar and skeletal chin dimensions associated with lower facial height among different divergent patterns. J Clin Diagn Res. 2016 May; 10(5):ZC75-80. https://doi.org/10.7860/JCDR/2016/19932.7811

12. Kim DK, Sung J, Song YM, Kim EM, Kim YH, Baek SH. Differences in heritability of craniofacial skeletal and dental characteristics between hypo- and hyper-divergent patterns using Falconer's method and principal component analysis. Angle Orthod. 2019 Mar;89(2):242-51. https://doi.org/10.2319/062518-475.1

13. Ajmera SN, Venkatesh S, Ganeshkar SV, Basalingappa S, Patil AK. Symphyseal angle : an angle to determine skeletal pattern using panoramic radiographs. Orthod Updat. 2014;7(October):137-9. https://doi.org/10.12968/ortu.2014.7.4.137

14. Steiner CC. The use of cephalometrics as an aid to planning and assessing orthodontic treatment. Report of a case. Am J Orthod. 1960;46(10):721-35. https://doi.org/10.1016/0002-9416(60)90145-7

15. Ahmed M, Shaikh A, Fida M. Diagnostic performance of various cephalometric parameters for the assessment of vertical growth pattern. Dental Press J Orthod. 2016 Jul-Aug;21(4):41-9. https://doi.org/10.1590/2177-6709.21.4.041-049.oar

16. Freudenthaler J, Čelar A, Ritt C, Mitteröcker P. Geometric morphometrics of different malocclusions in lateral skull radiographs. J Orofac Orthop. 2017 Jan;78(1):11-20. https://doi.org/10.1007/s00056-016-0057-x PMID:27796401

17. Sharma VK, Tandon P, Singh GK, Yadav K, Singh GP. Evaluation of skeletal patterns using panoramic radiography. J Adv Res Dent Oral Heal. 2017;2(1\&2):1-8. https://doi.org/10.24321/2456.141X.201701

18. Arnqvist G, Mårtensson T. Measurement error in geometric morphometrics: empirical strategies to assess and reduce its impact on measures of shape. Acta Zool Acad Sci Hung. 1998;44(1):73-96.

19. Klingenberg CP. Size, shape, and form: concepts of allometry in geometric morphometrics. Dev Genes Evol. 2016 Jun;226(3):113-37. https://doi.org/10.1007/s00427-016-0539-2

20. Mitteroecker P, Gunz P. Advances in Geometric morphometrics. Evol Biol. 2009;36(2):235-47. https://doi.org/10.1007/s11692-009-9055-x

21. Coogan JS, Kim DG, Bredbenner TL, Nicolella DP. Determination of sex differences of human cadaveric mandibular condyles using statistical shape and trait modeling. Bone. 2018 Jan;106:35-41. https://doi.org/10.1016/j.bone.2017.10.003

22. Klingenberg CP. MorphoJ: an integrated software package for geometric morphometrics. Mol Ecol Resour. 2011 Mar;11(2):353-7. https://doi.org/10.1111/j.1755-0998.2010.02924.x

23. MacLeod N. PalaeoMath: Part 25 - the centre cannot hold ii: elliptic fourier analysis. Palaeontol Newsl. 2012;79:29-43.

24. Bhullar MK, Uppal AS, Kochhar GK, Chachra S, Kochhar AS. Comparison of gonial angle determination from cephalograms and orthopantomogram. Indian J Dent. 2014 Jul;5(3):123-6. https://doi.org/10.4103/0975-962X.140820

25. Davies JC, Chan HH, Jozaghi Y, Goldstein DP, Irish JC. Analysis of simulated mandibular reconstruction using a segmental mirroring technique. J Craniomaxillofac Surg. 2019 Mar;47(3):468-72. https://doi.org/10.1016/i.jcms.2018.12.016

26. Motlagh MF, Bayat M, Naii S. Bone allograft: an option for total mandibular reconstruction. Craniomaxillofac Trauma Reconstr. 2017 Dec;10(4):306-13. https://doi.org/10.1055/s-0036-1593474

27. Rachmiel $A$, Shilo D, Blanc $O$, Emodi $O$. Reconstruction of complex mandibular defects using integrated dental custom-made titanium implants. Br J Oral Maxillofac Surg. 2017 May;55(4):425-7. https://doi.org/10.1016/i.bjoms.2017.01.006

28. Ferrario VF, Sforza C, Miani A Jr, Sigurtà D. Asymmetry of normal mandibular condylar shape. Acta Anat (Basel). 1997;158(4):266-73. https://doi.org/10.1159/000147939 